\title{
Gain-Scheduled Lateral Control of the F-14 Aircraft During Powered Approach Landing
}

\author{
Ian Fialho* and Gary J. Balas ${ }^{\dagger}$ \\ University of Minnesota, Minneapolis, Minnesota 55455 \\ Andrew K. Packard \\ University of California, Berkeley, California 94720 \\ and \\ Joseph Renfrow ${ }^{\S}$ and Chris Mullaney ${ }^{\mathrm{I}}$ \\ Naval Air Warfare Center, Patuxent River, Maryland 20670
}

\begin{abstract}
The design of a linear fractional transformation gain-scheduled controller, scheduled on angle of attack, for the F-14 aircraft lateral-directional axis is presented. The controller is designed for the powered approach flight phase, during which the angle of attack and corresponding airspeed varies from $2 \mathrm{deg}$ and $182 \mathrm{kn}$ to $14 \mathrm{deg}$ and $126 \mathrm{kn}$. A linear fractional model of the lateral dynamics is constructed based on four linearized models that correspond to 2, $\mathbf{6 , 1 0 . 5}$, and 14 deg angle of attack. Using parameter-dependent functions, a controller is designed that depends in a linear fractional manner on angle of attack and delivers uniform handling quality over angle-of-attack variations that lie between 2-14 deg. The resulting controller performs well when implemented in a nonlinear simulation model of the F-14 aircraft.
\end{abstract}

$p$
$r$
$v$
$y_{\text {ac }}$
$\alpha$
$\beta$
$\delta_{\text {dsp }}$
$\delta_{\text {dstab }}$
$\delta_{\text {stk }}$
$\delta_{\text {rud }}$
$\delta_{\text {rudp }}$
$\phi$

$=$ roll rate
$=$ yaw rate
$=$ lateral velocity
$=$ lateral acceleration
$=$ angle of attack
$=$ sideslip angle
$=$ differential spoiler deflection
$=$ differential stabilizer deflection
$=$ lateral stick input
$=$ rudder deflection
$=$ rudder pedal input
$=$ bank angle

I. Introduction

$\mathbf{L}$ ANDING an aircraft on an aircraft carrier requires precise control in both longitudinal and lateral directions during the entire powered-approach (PA) landing phase. The margin for error is extremely small because the tail hook of the aircraft must engage one of four arrestment wires spaced $40 \mathrm{ft}(12.2 \mathrm{~m})$ apart on the deck. The F-14 PA flight phase starts at about 3 miles $(4.8 \mathrm{~km})$ from the aircraft carrier. The pilot engages the PA flight control system at approximately $180 \mathrm{kn}$ and $2 \mathrm{deg}$ angle of attack. The objective is to capture and stay on a -3-deg glide path, at 10.5 deg angle of attack and airspeed of $137 \mathrm{kn}$. Under these conditions, assuming no ship motion, the F-14 aircraft will clear the ship by $11 \mathrm{ft}$ $(3.4 \mathrm{~m})$ and touchdown $1 \mathrm{~s}$ later with an impact velocity of $13 \mathrm{ft} / \mathrm{s}$ $(4 \mathrm{~m} / \mathrm{s})$ (Ref. 1). Lateral line-up is generally considered to be the most critical aspect of a carrier landing. ${ }^{2}$ Reducing the pilot workload required for lateral line-up corrections gives the pilot more time to concentrate on maintaining optimum angle-of-attack and glide-

Received 27 August 1997; revision received 3 September 1999; accepted for publication 6 September 1999. Copyright (C) 1999 by the authors. Published by the American Institute of Aeronautics and Astronautics, Inc., with permission.

${ }^{*}$ Postdoctoral Researcher, Department of Aerospace Engineering and Mechanics; currently Member of Technical Staff, Dynacs Engineering, Houston, TX.

Associate Professor, Department of Aerospace Engineering and Mechanics. Senior Member AIAA.

$\stackrel{+}{+}$ Professor, Department of Mechanical Engineering. Member AIAA

${ }^{\S}$ Engineer, Aircraft Division.

"Engineer, Aircraft Division. path angle. This improves the overall situational awareness of the pilot and consequently his ability to consistently land the aircraft on the first approach.

The originalF-14 aircraft was equipped with an analog flight control system (AFCS) to improve handling qualities in the pitch and lateral axis during a PA landing. The AFCS uses differential stabilizers in the feedback loop in addition to feedforward differential spoiler deflections to generate roll moments and rudder deflections in the feedback loop to generate sideslip. The location of these control surfaces on the F-14 aircraft is shown in Fig. 1. Even with this controller, there was significant coupling in the lateral-directiona axis, and this resulted in pilots having difficulty landing the F-14 aircraft on a carrier. More specifically, an AFCS equipped F-14 generates significantly large adverse sideslip in response to lateral stick inputs. This sideslip, coupled with the airplane's strong positive dihedral effect, tends to excite the Dutch-roll mode. This characteristic significantly degrades the pilot's ability to make accurate lateral line-up corrections during PA. The pilot is required to coordinate lateral stick inputs with rudder pedal inputs, and this detracts from his overall situational awareness.

In response to the AFCS deficiencies, in 1993 the U.S. Navy initiated a program to upgrade the AFCS to a digital flight control system (DFCS). ${ }^{3}$ The DFCS, designed by engineers from Grumman Aerospace and the Naval Air Warfare Center (NAWC), Patuxent River, uses a completely different PA controller configuration (see Ref. 4). Specifically, the roll axis is designed to be a roll-rate command system, which results in significant improvement in roll damping. To ensure that the PA DFCS is a "feet-on-the-floor" controller, lateral stick to rudder feedthrough, as well as sideslip rate feedback is used to reduce roll-sideslip cross coupling and to increase the damping of the Dutch-roll mode. The DFCS is designed using a classical loop-at-a-time approach. At each point in the PA phase feedback, gains are chosen using root-locus/frequencyresponse techniques. The gains are interpolated to obtain a gainscheduled controller for the PA phase. Based on pilot comments during test flights in 1995, the DFCS is a significant improvement over the AFCS as far as PA is concerned (see Refs. 2 and 3).

A paralleleffort to the DFCS program was initiated by the NAWC to design controllersfor the F-14 lateralaxis using modern multivariable control techniques. In Ref. 5 a linear controller was designed for an angle-of-attack/airspeed condition of $10.5 \mathrm{deg} / 137 \mathrm{kn}$, using the structured singular value $(\mu)$ framework. ${ }^{6-9}$ The desired rollrate and sideslip handling qualities were cast as model matching 

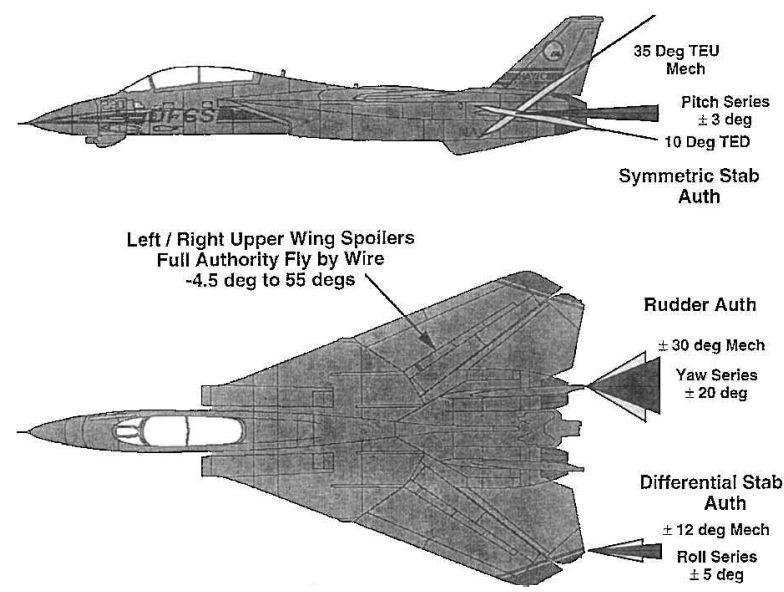

Fig. 1 F-14 aircraft control surfaces.

problems and solved using D-K iteration techniques. The configuration of the $\mu$ controller is significantly different from that of the DFCS. The $\mu$ controller uses the spoilers, rudders, and differential stabilizers in the feedback loop alone and does not use estimated sideslip rate as a control measurement. Unlike the piecewise linear response of the AFCS and DFCS, the $\mu$ controller has a linear response from stick to roll rate. This controller improves the roll-rate tracking and significantly lowers sideslip buildup during roll maneuvers. It outperformed the DFCS during on speed, i.e., $10.5 \mathrm{deg} / 137 \mathrm{kn}$, pilot-in-the-loop simulations at NAWC in 1995. The reader is referred to Ref. 5 for more details.

In this paper we build on the linear $\mu$-controller point design and present the design of a gain-scheduled controller, scheduled on angle of attack, that delivers uniform handling quality over the entire PA flight phase. The gain-scheduling paradigm used here is based on a linear fractional representation of the variation of the F-14 lateral dynamics with angle of attack. In Sec. II, the linear fractional transformation (LFT) approach to gain-scheduled design is described Ref. 10. The linear fractional modeling of the F-14 lateral dynamics, based on four linearized models that correspond to $\alpha=2,6,10.5$, and $14 \mathrm{deg}$ and corresponding airspeeds of 182 , 154,137 , and $126 \mathrm{kn}$, respectively, is discussed in Sec. III. The control design objective is to have lateral stick inputs command roll rate with a small amount of sideslip during a roll maneuver and for the rudder pedal inputs to command the sideslip angle.

In Sec. IV, a detailed discussion of the lateral handling quality requirements, the dynamic characteristics of the F-14 aircraft, and the control interconnection used to design the gain-scheduled controller is presented. The rationale behind the control interconnection and how the variation in dynamic behavior can be accounted for by using parameter-dependent functions is discussed in Sec. V. Controller analysis and nonlinear simulation results are the focus of Sec. VI. Pilot-in-the-loop simulation results for an earlier version of the gain-scheduledLFT controller is presented and compare with the redesigned gain-scheduledLFT controller. Concluding remarks are found in Sec. VII.

\section{Gain-Scheduling Based on LFT}

In this section we provide a brief overview of the linear fractional approach to gain scheduling. This approach makes use of the smallgain theorem, along with ideas from robustness theory for structured uncertainty. For more details the reader is referred to Ref. 10

Consider a parameter dependent plant $\boldsymbol{P}$ of a special structure: the linear fractional interconnection of a time-varying, blockstructured matrix $\Delta(t)$ and a linear time-invariant system $P$ as shown at the top of Fig. 2. The parameter dependence of the plant is caused by the varying $\Delta$ matrix. Typically, $\Delta$ is of the form $\Delta(t)=\operatorname{diag}\left[\delta_{1}(t) I_{r_{1}}, \delta_{2}(t) I_{r_{2}}, \ldots \delta_{f}(t) I_{r_{f}}\right]$, where $\delta_{1}(\cdot), \ldots, \delta_{f}(\cdot)$ are exogenoustime-varying parameters upon which the dynamics of the plant depend. As far as the F-14 lateral dynamics during landing is concerned, the only time-varying parameter is angle of attack, and hence in our case $\Delta(t)=\alpha(t) I_{r}$. The assumption is made that the

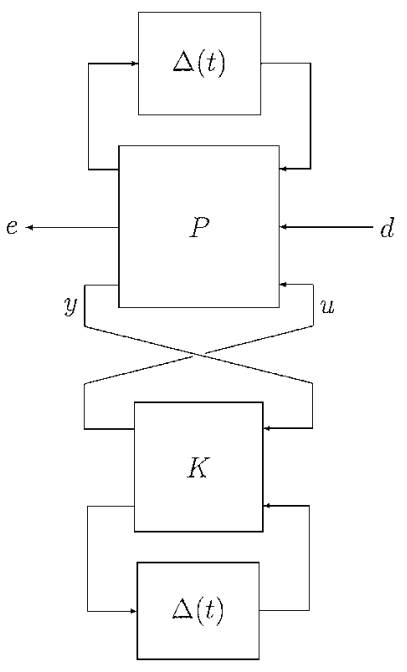

Fig. 2 LFT closed-loop system.

value of $\Delta(t)$ is guaranteeda priori to lie in a known bounded set $B \Delta$, e.g., $B \Delta=\{\Delta:\|\Delta\| \leq 1\}$ and the exact value $\Delta(t)$ can be measured in real time. Hence the controller may use this real-time knowledge of the time-varying parameter. The controller is restricted to have a similar structure to that of the plant: a linear fractional interconnection of an linear time invariant (LTI) system $K$ with a copy of $\Delta(t)$ that affects the gain scheduling. This controller structure is shown at the bottom of Fig. 2.

Note that the parameter-dependentcontroller can actually be implemented in two different manners: first, exactly as shown, as a linear time-invariant part $K$ running on a digital signal processor with the gain scheduling being affected entirely by the linear-fractional feedback around the bottom channels; or it can be implemented by programming the appropriate time-varying differentialequations for the operator $K_{11}+K_{12} \Delta(t)\left[I-K_{22} \Delta(t)\right]^{-1} K_{21}$. In any case, by interconnecting the parameter-dependentplant with the parameterdependent controller, the closed-loop system appears as in Fig. 2. Note that the closed-loop system depends on multiple copies of the parameter $\Delta$

Drawn in this manner, the closed-loop system appears as a finite dimensional,LTI system, i.e., the interconnection of $P$ and $K$, subjected to a time-varying perturbationmade up of a repeated structure. The perturbation consists of two parts: the physical parameter $\Delta(t)$, which affects the plant, and the sensed value of $\Delta(t)$, which is used in the controller. These together give rise to the repeated structure. The control objective is to design $K$ such that, for all allowable parameter trajectories $\Delta(t)$, the parameter-dependent closed-loop system is internally exponentially stable and has a small induced $\mathcal{L}_{2}$ norm from $d$ to $e$. The stability of the closed-loop system and the worst-case $\mathcal{L}_{2}$ norm of the $d \rightarrow e$ channel, over allowable timevarying $\Delta(t)$, can be bounded using a scaled, small-gain theorem. The scaling matrices are restricted to be constant, diagonal matrices that commute with the repeated structure of the perturbation. These scaling matrices are typical of robustness analysis to structured uncertainty. The main result is that the existence of a controller satisfying the scaled small-gain bound can be expressed exactly as the feasibility of a finite dimensional, convex program, in particular an affine matrix inequality. The reader is referred to Ref. 10 for the exact inequalities. Furthermore, if there is no parameter dependence, i.e., just a standard LTI problem, the bound on $d \rightarrow e$ is the $\mathcal{H}_{\infty}$ norm, and the conditions reduce to the well-known necessary and sufficient conditions for the existence of controllers achieving a prespecified level of $\mathcal{H}_{\infty}$ disturbance rejection. So the LFT formulation is a parameter-dependentcontrol synthesis problem, which is a generalization of the $\mathcal{H}_{\infty}$ optimal control problem.

\section{Linear Fractional Modeling of the F-14}

The modeling of the F-14 dynamics in linear fractional form, as shown in the top of Fig. 2, is presented in this section. This model is constructed from four linearized models that correspond to $\alpha=2,6,10.5$, and $14 \mathrm{deg}$ and corresponding airspeeds of 182 , 154,137 , and $126 \mathrm{kn}$, respectively. The linear models were obtained 
by using the linear model extraction tool developed at NAWC, on the nonlinearmodel of the F-14 aircraft (see Ref. 4 for more details). Each of these linear models have three control inputs- $\delta_{\text {dstab }}, \delta_{\text {rud }}$, and $\delta_{\mathrm{dsp}}$; five outputs $-\beta, p, r, \phi$, and $y_{\mathrm{ac}}$; and four states $-v, r, p$, and $\phi$. The linearized models may be found in Ref. 11 (p. 11). The underlying assumption here is that the time-varying dependence of the F-14 dynamics on $\alpha$ can be represented by a parameterdependent state-space description as follows:

$$
\left[\begin{array}{c}
\dot{v} \\
\dot{r} \\
\dot{p} \\
\dot{\phi} \\
\beta \\
p \\
r \\
\phi \\
y_{\mathrm{ac}}
\end{array}\right]=\left[\begin{array}{cc}
A(\alpha) & B(\alpha) \\
C(\alpha) & D(\alpha)
\end{array}\right] \cdot\left[\begin{array}{c}
v \\
r \\
p \\
\phi \\
\delta_{\mathrm{dstab}} \\
\delta_{\mathrm{rud}} \\
\delta_{\mathrm{dsp}}
\end{array}\right]:=M(\alpha) \cdot u
$$

Using polynomial interpolation on the four linearized models, i.e., on $M(2), M(6), M(10.5), M(14)$, we approximate $M(\alpha), \alpha$ in degrees, as

$$
M(\alpha)=M_{0}+M_{1} \cdot \alpha+M_{2} \cdot \alpha^{2}+M_{3} \cdot \alpha^{3}
$$

Because we have fourinterpolationpoints, a third-orderexpansion is exact. The matrices $M_{0}, M_{1}, M_{2}$, and $M_{3}$ are listed in the Appendix. For more details on modeling of parametric uncertainty via LFTs, see Refs. 12-14.

We now convert $M(\alpha)$ into a linear fractional transformation form. If $r_{i}$ is the highest interpolating polynomial degree in the $i$ th row of $M(\alpha)$, then this row can be written as

$$
\begin{aligned}
M_{i}(\alpha) & =\left[M_{i}^{0}+M_{i}^{1} \alpha+\cdots+M_{i}^{r_{i}} \alpha^{r_{i}}\right] \\
& =M_{i}^{0}+\left[\alpha \cdots \alpha^{r_{i}}\right] \cdot\left[\begin{array}{c}
M_{i}^{1} \\
\cdot \\
M_{i}^{r_{i}}
\end{array}\right]
\end{aligned}
$$

The term $\left[\alpha \cdots \alpha^{r_{i}}\right]$ in the preceding equation is a simple rank $r_{i}$ LFT on $\alpha$, and hence the $i$ th row may be drawn as shown in Fig. 3 .

Once each row of $M(\alpha)$ has been represented in the preceding form, it is a simple task to convert $M(\alpha)$ into linear fractional form, for example, by using the $\mu$-Analysis and Synthesis Toolbox ( $\mu$-Tools) command sysic. ${ }^{7}$ We shall denote the time-invariant block of the F-14 linear fractional model as F14 nom. The variable $r$, i.e., the total number of $\alpha$ copies, is given as

$$
r=\sum_{i=1}^{9} r_{i}
$$

In our case $r=13$.

\section{Requirements and Controller Architecture}

The objective is to design a controller, scheduled on $\alpha$, that delivers uniform performance over all possible time variations of $\alpha$ that lie between 2 and $14 \mathrm{deg}$. This implies that there are no rate limits on $|\dot{\alpha}|$ in the control design which may lead to conservative results. The assumption is made that the controller measures $\alpha, \delta_{\text {stk }}$, $\delta_{\text {rudp }}, p, r$, and $y_{\text {ac }}$. The controller generates commands to the stabilizers, rudders, and spoilers. Performance is defined in terms of a desired handling quality (HQ) response from the pilot's lateral stick input to roll rate and from the rudder pedals to sideslip angle. It is also desired that the controller have a prescribed level of robustness to uncertainty in the lateral dynamics, e.g., uncertainty

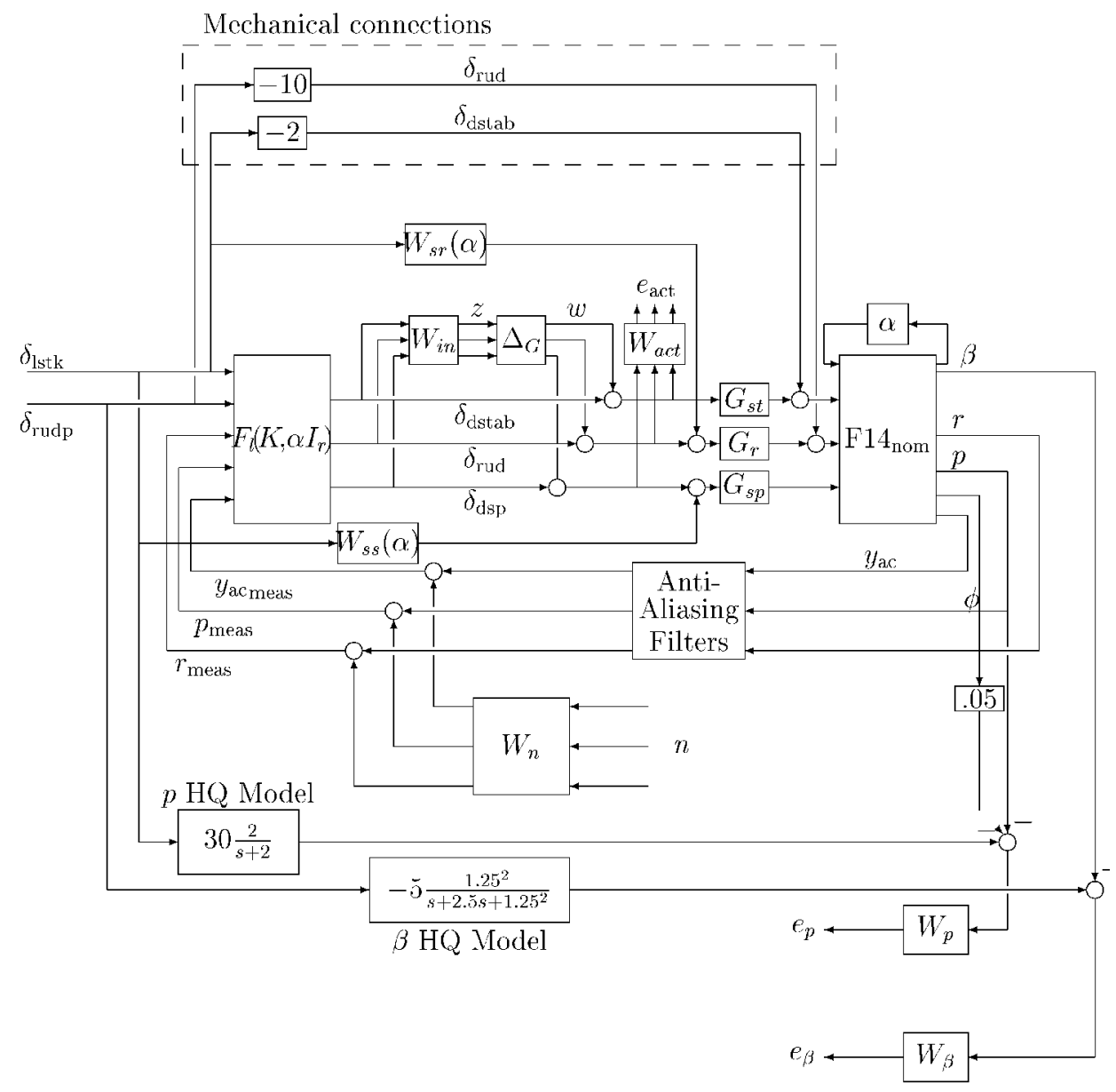

Fig. 3 Representation of the $i$ th row of $M(\alpha)$. 


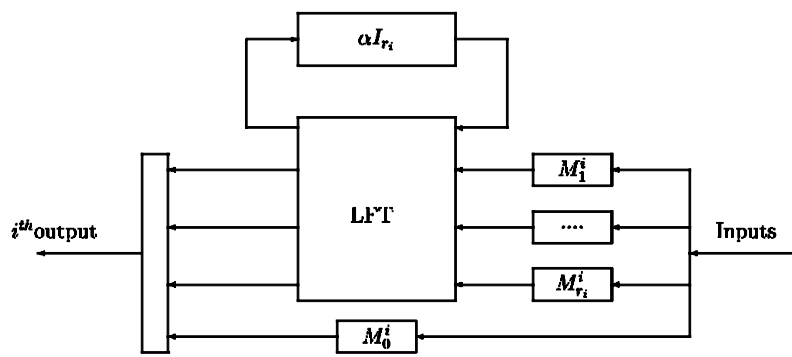

Fig. 4 LFT design interconnection structure.

in deflection to force/moment ratios, unmodeled dynamics such as fuel slosh and flexible modes, etc. As in standard $\mathcal{H}_{\infty} / \mu$ design these performance and robustness objectives are accounted for by using frequency-dependent weighting functions. Because we are designing a controller that varies as a function of $\alpha$, in addition to being frequency dependent the weighting functions may be chosen to be parameter $(\alpha)$ dependent as well. This additional degree of freedom allows us to compensate for the variation in aircraft dynamics across the PA phase and plays an important role in the design process. The choice of these weighting functions will be discussed in the next two sections. A block diagram of the interconnection structure for the F-14 lateral control design is shown in Fig. 4.

The stabilizer actuators, denoted as $G_{\text {st }}$ in Fig. 4, are modeled as a first-order system $25 /(s+25)$ and have \pm 12 deg and $\pm 36 \mathrm{deg} / \mathrm{s}$ deflection and deflection rate limits. The series servos driving these actuators from the fly-by-wire control system have $\pm 5 \mathrm{deg}$ and $\pm 33 \mathrm{deg} / \mathrm{s}$ deflection and deflection rate limits, respectively. The stabilizers operate in a differential manner. The rudder actuators, denoted as $G_{r}$ in Fig. 4, are modeled as a first-order system $25 /(s+25)$. These actuators have $\pm 30 \mathrm{deg}$ and $\pm 125 \mathrm{deg} / \mathrm{s}$ deflection and deflection rate limits. The series servos driving these actuators from the fly-by-wire control system have $\pm 19 \mathrm{deg}$ and $\pm 126 \mathrm{deg} / \mathrm{s}$ deflection and deflection rate limits, respectively.

The F-14 aircraft has four spoiler panels per wing. They are commanded in pairs, resulting in four effective control surfaces. All four pairs can be deflected differentially for roll control and the inboard pairs deflected symmetrically for direct lift control. For the present design the four spoiler control surfaces are ganged together resulting in a single, differential spoiler command for roll. Each spoiler can be deflected through a range of $-4.5-55.0 \mathrm{deg}$. The total deflection range is therefore $59.5 \mathrm{deg}$. Once on the glide path, the assumption is made that the spoiler panels are trimmed at $+25 \mathrm{deg}$, allowing for approximately \pm 30 deg of deflection about trim. The spoiler actuators $G_{\mathrm{sp}}$, driven directly by the fly-by-wire control system, are modeled as a first-order system $250 /(s+250)$ and have a $\pm 250 \mathrm{deg} / \mathrm{s}$ deflection rate limit.

Roll rate, yaw rate, and lateral acceleration measurements are available for feedback. The roll-rate gyro is modeled as a secondorder system $157^{2} /\left(s^{2}+220 s+157^{2}\right)$ and measure rates up to $\pm 250 \mathrm{deg} / \mathrm{s}$. The yaw-rate gyro is modeled as a second-order system $137^{2} /\left(s^{2}+193 s+137^{2}\right)$ and measure rates up to $\pm 50 \mathrm{deg} / \mathrm{s}$. The lateral accelerometer is modeled as a second-order system $315^{2} /\left(s^{2}+440 s+315^{2}\right)$. This accelerometer can measure acceleration up to $\pm 1 \mathrm{~g}$.

The three measurement signals, roll rate, yaw rate, and lateral acceleration, are passed through second-order, antialiasing filters prior to being fed to the controller. The natural frequency and damping values for the yaw rate and lateral acceleration filters are $12.5 \mathrm{~Hz}$ and 0.5 , respectively, whereas these values for the roll-rate filter are $4.1 \mathrm{~Hz}$ and 0.7 . The antialiasing filters have unity gain at direct current.

The performance objective is to have the aircraft respond effectively to the pilot's lateral stick and rudder pedal inputs. These are listed next and correspond to Level One Handling Qualities as defined in the military flying quality specifications MIL-F-8785C:

1) The aircraft HQ response from the lateral stick to the roll rate should correspond to that of a first-order system $30[2 /(s+2)]$ $\operatorname{deg} / \mathrm{s} / \mathrm{in}$.
2) The aircraft HQ response from the rudder pedals to sideslip angle should correspond to $-5\left[1.25^{2} /\left(s^{2}+2.5 s+1.25^{2}\right)\right] \mathrm{deg} / \mathrm{in}$.

Objective 1 is of great importance because lateral line-up corrections are made with the lateral stick alone, and hence it is important that this response be well damped and highly predictable. In addition, it is desired that only a small amount of sideslip be generated in response to lateral stick commands. In principle, small sideslip, whether of the same sign as the roll rate or of opposite sign, can seem equally acceptable. However, our experience with pilot-in-the-loop testing of earlier versions of the present controller indicates that this is certainly not true from the pilots' perspective. During a roll maneuver, there are two notions of sideslip directionality: adverse (same sign as the roll rate or nose outside the turn) and proverse (sign opposite to the roll rate or nose inside the turn). If the sideslip generated is proverse, the nose of the aircraft leads into a turn. This requires the pilot to lead the heading angle by a few degrees, and most pilots find this annoying. On the other hand, most pilots find a small amount of adverse sideslip (nose lagging) a natural consequence of a roll maneuver. Thus the directionality of the regulated variable needs to be considered $-a$ feature that is not discerned by norm-based design techniques. The situation is even more complicated in our case because the F-14 aircraft has a natural proverse tendency at low angles of attack, i.e., at 2-6 deg, whereas at high angles of attack the natural sideslip direction is adverse. Because of the preceding reasons, we found it necessary to use parameter-dependentfeedthrough connections between the stick and spoilers/rudders $\left[W_{\mathrm{ss}}(\alpha)\right.$ and $W_{\mathrm{sr}}(\alpha)$ in Fig. 4], to ensure uniform sideslip directionality and magnitude during PA. This ensures that the PA controller is a true feet-on-the-floor controller and obviates the necessity of stick/rudder coordination during the landing phase. The preceding argument also serves to demonstrate the fact that the control problem considered here truly requires a gainscheduled solution and that a single robust linear controller would be a significant compromise. From the pilot's perspective Objective 2 may be relaxed considerably. The reason for this is that the pilot uses the rudder pedals primarily to null out crosswinds and that under normal conditions the PA control system is a feet-on-the-floor control system. Hence, in our design we shall consider Objective 2 to be of secondary importance.

\section{Weighting Functions}

The selection of the various components in Fig. 4 are presented in this section. The variation in lateral dynamics of the F-14 during landing and illustration of how this is compensated for by the use of parameter-dependentfunctions is also discussed.

\section{A. HQ Weighting Functions}

The desired $\delta_{\text {stk }}$-to- $p$ response is cast as a model-matching problem as shown in Fig. 4. The $W_{p}$ weight that penalizes the difference between the ideal roll-rate response and the actual roll-rate response is $W_{p}=[0.0240(s+180.48) /(s+1.875)]$. This weight implies a desired tracking accuracy of $0.5 \mathrm{deg} / \mathrm{s}$ at low frequency that is relaxed to $5 \mathrm{deg} / \mathrm{s}$ at $20 \mathrm{rad} / \mathrm{s}$. A magnitude plot of $W_{p}$ is shown in Fig. 5. Observe that a small amount of bank angle $\phi$ has been blended with the roll-rate error. The purpose of this blending is to eliminate the effect of an open-loopright-half plane zero associated with the lateral stick to roll-rate transfer function. This results in the lateral stick to blended roll rate and bank angle being minimum phase while retaining the general transfer function response of the lateral stick to roll rate between 0.1 and $20 \mathrm{rad} / \mathrm{s}$. The benefit of this modification is that the weighting function $W_{p}$ can be chosen to be of first order.

The desired $\delta_{\text {rudp }}$-to- $\beta$ response is also cast as a model-matching problem. The $W_{\beta}$ weight that penalizes the difference between the ideal sideslip response and the actual sideslip response to $\delta_{\text {rudp }}$ is chosen to be $W_{\beta}=0.8$, which correspondto allowable tracking error of $1.25 \mathrm{deg}$.

\section{B. Parameter-Dependent Weights}

Parameter-dependent functions $W_{\mathrm{ss}}(\alpha)$ and $W_{\mathrm{sr}}(\alpha)$ are incorporated into the control design interconnection shown in Fig. 4 to compensate for the variation in sideslip directionality during roll 


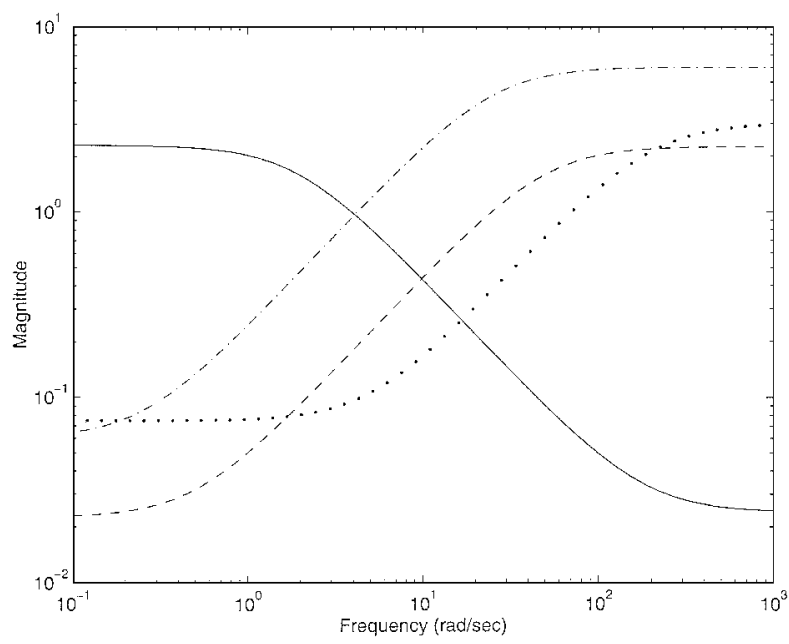

Fig. 5 Roll-rate error weight $W_{p}(-), W_{\text {in }}$ stabilizer weight (- - -), $W_{\text {in }}$ rudder weight $(-\cdot)$, and $W_{\text {in }}$ spoiler weight $(\cdots)$.
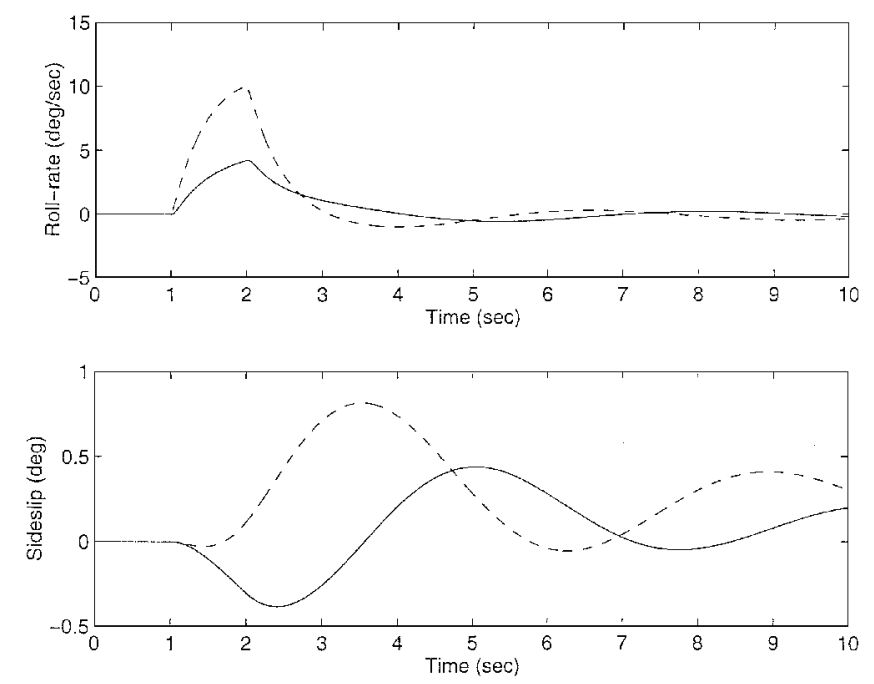

Fig. 6 - 2-deg stabilizer pulse response with 0-deg spoiler deflection (—), and - 2-deg stabilizer with a $-6 \bowtie-d e g$ spoiler pulse response $(--)$ at $\alpha=2$ deg.

maneuvers. These functions represent constraints on the direct feedthrough structure of the LFT controller. Thus $W_{\mathrm{ss}}(\alpha)$ corresponds to a direct coupling between the lateral stick input and the spoiler command, whereas $W_{\mathrm{sr}}(\alpha)$ corresponds to a direct coupling between the lateral stick input and the rudder command. The openloop interconnection that is used to design the controller $F_{l}(K, \alpha)$ includes these direct coupling functions. The final LFT controller is constructed by absorbing $W_{\mathrm{ss}}(\alpha)$ and $W_{\mathrm{sr}}(\alpha)$ into $F_{l}(K, \alpha)$. If $W_{\text {in }}$ and $W_{\text {act }}$ are chosen appropriately, the direct feedthrough terms of this controller will be close to those defined by $W_{\mathrm{ss}}(\alpha)$ and $W_{\mathrm{sr}}(\alpha)$.

To understand the rationale behind $W_{\mathrm{ss}}(\alpha)$, we first consider the open-loop responses at $\alpha=2 \mathrm{deg}$, as shown in Fig. 6 . The solid responses correspond to a $-2 \mathrm{deg} / 1 \mathrm{~s}$ stabilizer pulse. As can be seen, a significant amount of proverse sideslip is generated because of this stabilizer deflection. Figure 6 serves to illustrate that rapid stabilizer deflections are the main cause of the proverse sideslip tendency at low angles of attack. Unfortunately, stabilizer deflection in response to a stick command cannot be avoided because there is a direct mechanical connection between the stick and differential stabilizer, with a gain of 2 (see Fig. 4). The dashed responses in Fig. 6 correspond to a $-6.1 \mathrm{deg}, 1-\mathrm{s}$ spoiler pulse applied simultaneously with the preceding stabilizer pulse. Observe that the proverse sideslip is significantly reduced by the addition of the spoiler deflection. The adverse sideslip tendency in response to the spoiler deflection nullifies the proverse tendency caused by the
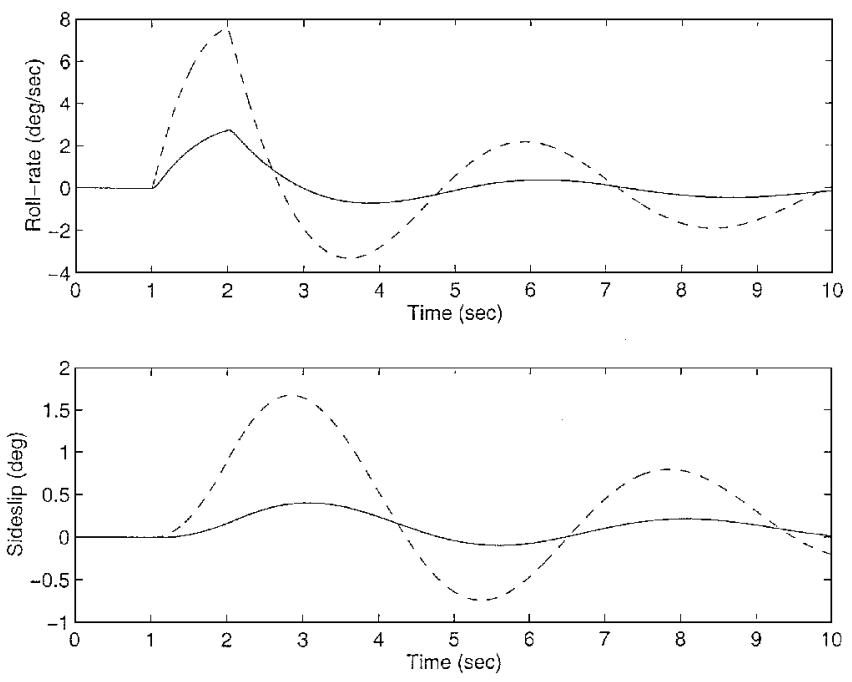

Fig. 7 -2-deg stabilizer pulse response with 0-deg spoiler deflection (- , and - 2-deg stabilizer with a $-6 \bowtie-$ deg spoiler pulse response $(---)$ at $\alpha=10.5 \mathrm{deg}$.

stabilizer deflection. Because of this fact, a direct feedthrough from the stick to the spoiler is included to match the stabilizer mechanical connection. Figure 7 shows responses to the preceding inputs at $\alpha=10.5 \mathrm{deg}$. Observe that the stabilizer deflection does not cause any proverse sideslip at this flight condition, although the amount of adverse sideslip is much larger. Because of this reason, we choose the stick-to-spoiler feedthrough to be parameter dependent. More specifically, we choose $W_{\mathrm{ss}}(\alpha)$ to be larger at smaller $\alpha$. In linear fractional form $W_{\mathrm{ss}}(\alpha)$ is chosen to be

$$
W_{\mathrm{ss}}(\alpha)=F_{u}\left(\left[\begin{array}{cc}
0 & 0.5083 \\
1 & -7.1167
\end{array}\right], \alpha\right)=-7.1167+0.5083 \alpha
$$

In addition to the preceding stick-spoilerfeedthrough, we impose a stick-rudder feedthrough as well. The feedthrough $W_{\mathrm{sr}}(\alpha)$ is once again parameter dependent and given by

$$
W_{\mathrm{sr}}(\alpha)=F_{u}\left(\left[\begin{array}{cc}
0 & -1.7647 \\
1 & 8.5294
\end{array}\right], \alpha\right)=8.5294-1.7647 \alpha
$$

At low $\alpha W_{\text {sr }}(\alpha)$ is positive, and this supplements the spoiler feedthrough in ensuring that the sideslip generated is adverse. At high $\alpha W_{\text {sr }}(\alpha)$ is large and negative to counter the large adverse sideslip tendency at this flight condition. The reason why $W_{\mathrm{sr}}(\alpha)$ is so large at high $\alpha$ is explained next.

Remark 1: The current $\mathrm{DFCS}^{4}$ uses similar feedthrough terms, which is the reason why the DFCS is called the PA-automatic rudder interconnect.

\section{Actuator Magnitude Constraints}

Limits on the actuator deflection magnitudes are accounted for by the weight $W_{\text {act }}$. $W_{\text {act }}$ is a $3 \times 3$ diagonal matrix given by

$$
W_{\text {act }}=\operatorname{diag}(7.2,1.11,0.0037)
$$

The weight on the stabilizerdeflection, i.e., $W_{\mathrm{act}}^{11}$, is relatively high in order to limit the stabilizer deflection to be twice (mechanical connection gain) the lateral stick deflection. This choice is dominated by the fact that at low angles of attack larger stabilizer deflections cause larger proverse sideslip, as illustrated in Fig. 6. The rudderdeflection weight, i.e., $W_{\text {act }}^{22}$, is also relatively high. This choice is dominated by the fact that at low angles of attack large negative rudder commands generated by the controller $F_{l}\left(K, \alpha I_{r}\right)$ nullify the effect of the stick-spoiler/stick-rudder feedthrough just described. Hence, it is necessary to limit the rudder commands generated by $F_{l}\left(K, \alpha I_{r}\right)$ at this flight condition. Unfortunately, the large weight $W_{\text {act }}^{22}$ limits the rudder commands generated by $F_{l}\left(K, \alpha I_{r}\right)$ at high angles of attack as well, resulting in large adverse sideslip at these flight conditions. This explains why we choose the stick-rudder feedthrough 
$W_{\text {sr }}(\alpha)$ to be large and negative at high $\alpha$. This choice results in larger rudder deflections at high $\alpha$ and helps limit adverse sideslip buildup at these flight conditions.

Remark 2: In the DFCS the amount of rudder deflection in response to a lateral stick input is relatively small, uniformly during PA. Although this serves to limit the proverse sideslip at low $\alpha$, the adverse sideslip buildup is significantly higher than in the present design.

\section{Uncertainty Modeling}

Uncertainty in the model of the F-14 aircraft is parameterized by the blocks $W_{\text {in }}$ and $\Delta_{G}$ shown in Fig. 4 . For the present design we choose not to model uncertainty in a detailed manner, but rather to lump all types of model uncertainty together into three individual, complex, multiplicative uncertainties at the input of the aircraft nominal model. Hence, $\Delta_{G}=\operatorname{diag}\left(\Delta_{\text {dstab }}, \Delta_{\text {rud }}, \Delta_{\text {dsp }}\right)$ and $W_{\text {in }}=\operatorname{diag}\left(w_{\text {dstab }}, w_{\text {rud }}, w_{\text {dsp }}\right)$. Modeling the uncertainty as three individual blocks is based on the assumption that isolated errors in the differential stabilizer, rudder, and differential spoiler channels do not couple to other channels. The transfer function $W_{\text {in }}$ is assumed known and reflects the amount of uncertainty in the model The transfer function $\Delta_{G}$ is assumed to be stable and unknown, except for the norm condition $\left\|\Delta_{G}\right\|_{\infty} \leq 1$. The input uncertainty was modeled as

$$
W_{\text {in }}=\operatorname{diag}\left[\frac{2.25(s+0.5)}{s+50}, \frac{6(s+0.25)}{s+25}, \frac{3(s+5)}{s+200}\right]
$$

A frequency plot of $W_{\text {in }}$ is in Fig. 5. At any frequency the magnitude of the weight can be interpreted as the percentage of uncertainty in the model at that frequency. An alternate interpretation of the weight $W_{\text {in }}$ is that it serves to limit the bandwidth as measured at the actuators.

In the nonlinear simulation model the differential stabilizer and rudder moment coefficients are linear with respect to deflections. Hence the low-frequency uncertainty associated with the stabilizer (dashed line in Fig. 5) is small, i.e., 2.25\%. This rolls up to $225 \%$ at high frequency in order to restrict the stabilizer loop bandwidth. Similarly, $w_{\text {rud }}$ is $6 \%$ at low frequency and rolls up to $600 \%$ at high frequency in order to limit the rudder loop bandwidth. The differential spoilers are nonlinear with respect to spoiler deflection. The spoiler uncertainty is modeled as $7.5 \%$ at low frequency and $300 \%$ at high frequency.

We also include the noise weight $W_{n}=\operatorname{diag}(0.1,0.1,0.02)$ to model measurement noise in the three sensor channels. To reduce the state dimension of the controller, all of the actuators, i.e., $G_{\mathrm{st}}, G_{r}, G_{\mathrm{sp}}$, and gyros are modeled as unity in the present design. The neglected fast dynamics of the actuators and sensors are accounted for in the uncertainty weight $W_{\text {in }}$. Also, only the roll-rate antialiasing filter is included in the design, the filters in the other channels being modeled as unity.

The control interconnection diagram of Fig. 4 is converted to the standard form of Fig. 2. In our case $e$ and $d$ are

$$
e=\left[\begin{array}{c}
z \\
e_{p} \\
e_{\beta} \\
e_{\mathrm{act}}
\end{array}\right], \quad d=\left[\begin{array}{c}
w \\
n \\
\delta_{\text {Istk }} \\
\delta_{\text {rudp }}
\end{array}\right]
$$

Note that the control interconnection depends on 15 copies of the parameter $\alpha$, i.e., 13 from the F-14 LFT model and 2 from $W_{\mathrm{ss}}$ and $W_{\text {sr }}$.

\section{LFT Controller Analysis}

The LFT control synthesis algorithm, with the precedinginterconnection model as input data, returned an optimal $\gamma$ value of 42.89 . The resulting LFT controller $F_{l}\left(K, \alpha I_{r}\right)$ has 13 states. Because the control interconnection (see Fig. 4) depends on $15 \alpha$ copies, $F_{l}\left(K, \alpha I_{r}\right)$ has 15 copies as well, i.e., $r=15$. The final LFT controller is constructed by absorbing $W_{\mathrm{ss}}$ and $W_{\mathrm{sr}}$ into $F_{l}\left(K, \alpha I_{r}\right)$.
Table 1 Gain and phase margins, $\alpha=10.5 \mathrm{deg}$

\begin{tabular}{lcccc}
\hline \hline $\begin{array}{l}\text { Loop } \\
\text { broken }\end{array}$ & $\begin{array}{c}\mathrm{GM}, \\
\mathrm{dB}\end{array}$ & $\begin{array}{c}\omega_{\mathrm{GM}}, \\
\mathrm{rad} / \mathrm{s}\end{array}$ & $\begin{array}{c}\mathrm{PM}, \\
\mathrm{deg}\end{array}$ & $\begin{array}{l}\omega_{\mathrm{PM}}, \\
\mathrm{rad} / \mathrm{s}\end{array}$ \\
\hline$p$ & 15.83 & 17.9 & 64.83 & 4.318 \\
$r$ & 26.1 & 8.846 & 111 & 1.386 \\
$y_{\mathrm{ac}}$ & 38.11 & 2.061 & $\infty$ & $\mathrm{NaN}$ \\
$\delta_{\text {dstab }}$ & 43.31 & 23.65 & $\infty$ & $\mathrm{NaN}$ \\
$\delta_{\text {rud }}$ & 26.94 & 8.758 & 112.5 & 1.143 \\
$\delta_{\text {dsp }}$ & 16.03 & 17.65 & 65.11 & 4.246 \\
\hline \hline
\end{tabular}

\begin{tabular}{lcccc} 
Table 2 & \multicolumn{4}{c}{ Gain and phase margins, $\boldsymbol{\alpha}=\mathbf{2 ~ d e g}$} \\
\hline \hline $\begin{array}{l}\text { Loop } \\
\text { broken }\end{array}$ & $\begin{array}{c}\mathrm{GM}, \\
\mathrm{dB}\end{array}$ & $\begin{array}{c}\omega_{\mathrm{GM}}, \\
\mathrm{rad} / \mathrm{s}\end{array}$ & $\begin{array}{c}\mathrm{PM}, \\
\mathrm{deg}\end{array}$ & $\begin{array}{c}\omega_{\mathrm{PM}}, \\
\mathrm{rad} / \mathrm{s}\end{array}$ \\
\hline$p$ & 18.64 & 21.04 & 83.93 & 3.073 \\
$r$ & 19.5 & 0.3336 & 91.22 & 1.91 \\
$y_{\mathrm{ac}}$ & 30.72 & 2.157 & $\infty$ & $\mathrm{NaN}$ \\
$\delta_{\mathrm{dstab}}$ & 37.83 & 24.51 & $\infty$ & $\mathrm{NaN}$ \\
$\delta_{\text {rud }}$ & 23.47 & 9.545 & 98.12 & 1.643 \\
$\delta_{\mathrm{dsp}}$ & 19.43 & 20.87 & 83.89 & 3.078 \\
\hline \hline
\end{tabular}

Hence this controller depends on 17 copies of $\alpha$. In addition to performing well in the nonlinear simulation, it is required that the LFT controller frozen at the four angles of attack possess adequate robustness properties. The following section describes the frequencyresponse analysis of the LFT controller.

\section{A. Robustness Analysis}

To meet the loop-at-a-time robustness criterion of MIL-F-9490, each loop is required to have at least a 6-dB gain margin and a 45-deg phase margin. In addition, the bandwidth of the controlloops should be small to avoid exciting the unmodeled flexible modes of the aircraft. The closed-loop system used in the frequency-response computations includes the series servo, the actuator models, the frozen F-14 aircraft and controller model, the sensor models, and the antialiasing filters. In Table 1 we list the gain/phase margins (GM, PM) and the corresponding gain/phase crossover frequencies of the individual sensor and actuator loops broken a loop at a time, with the $\alpha$ value frozen at $10.5 \mathrm{deg}$. Table 1 is representative of the robustness margins at high angles of attack. Observe the relatively low bandwidths caused by the roll up of the uncertainty weight $W_{\text {in }}$, and the fact that the gain/phase margin specifications have been met or exceeded for all feedback loops. In Table 2 we list the gain/phase margins with $\alpha$ frozen at $2 \mathrm{deg}$. This table is representative of the robustness margins at low angles of attack. Once again bandwidth and gain/phase margin requirements are met or exceeded.

\section{B. Nonlinear Simulation}

We now turn our attention to the nonlinear simulation results for the LFT controller. All of the responses were generated with a SIMULINK-based nonlinear simulation model of the F-14 aircraft (see Ref. 4). This simulation model was developed at NAWC and is a very close approximation to the full-order FORTRAN nonlinear simulation. Unless otherwise stated, all responses correspond to the time-varying $\alpha$ trajectory shown in Fig. 8. This trajectory is chosen to be representative of the $\alpha$ variation during the landing phase. In all of the responses, the stick input is a 1.0 -in. $(2.5 \mathrm{~cm})$ doublet pair as shown in Fig. 8 filtered through the command shaping filter $W_{\text {ref }}(s)=2 \cdot 2 \cdot \pi /(s+2 \cdot 2 \cdot \pi)$. The rudder input is 0.5 times the stick input.

In Figs. 9 and 10 we show the $p, \phi, r, \beta$ responses, control surface deflections, and deflection rates to the lateral stick doublet pair. From these we see that the roll response is very close to the HQ model and is uniform over the range of $\alpha$. There is no proverse sideslip buildup, and moreover the sideslip response during a roll maneuver is uniform during PA. Because of the parameter-dependentfeedthrough terms that were incorporated in the control interconnection. The effect of these terms can be clearly seen from the control deflections plot of Fig. 10. Observe that the rudder deflections are significantly larger at high $\alpha$, whereas at low $\alpha$ the rudder has a small kickback 

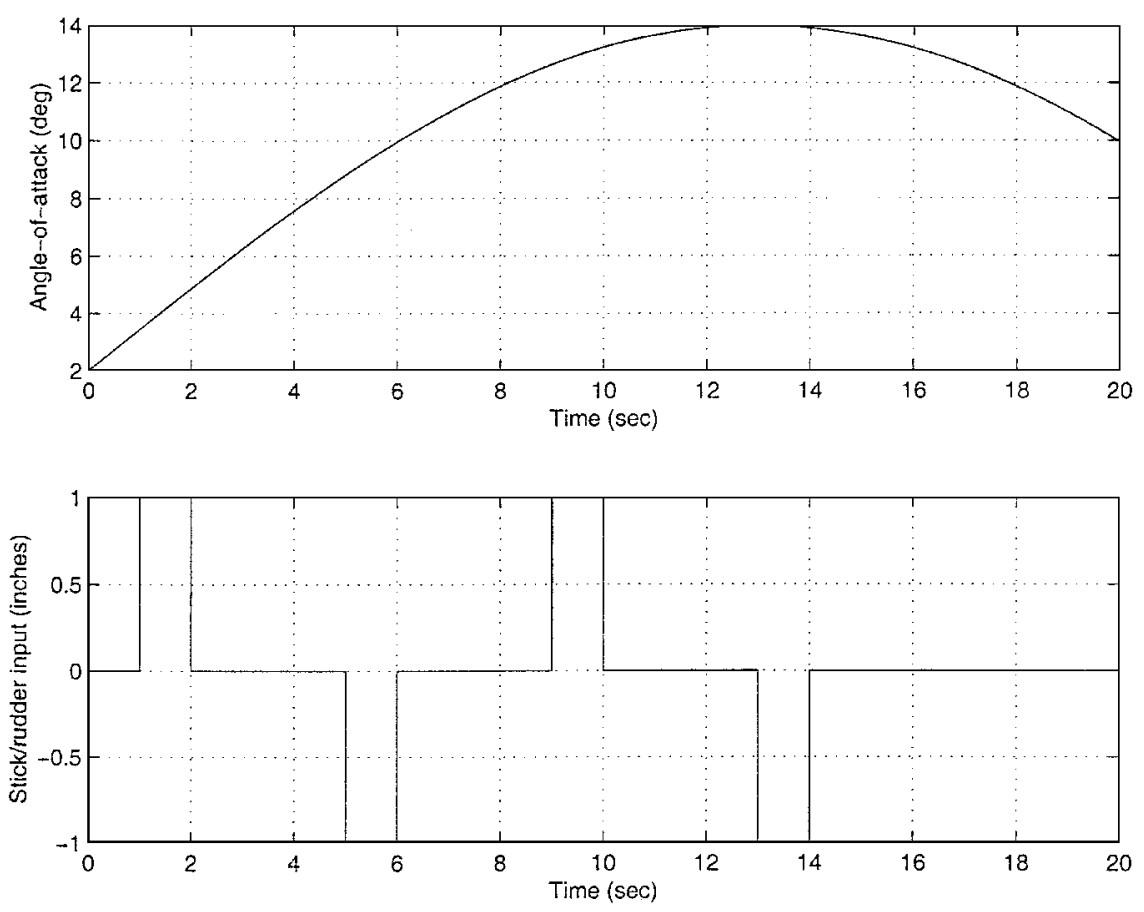

Fig. $8 \alpha$ trajectory and stick/rudder input.
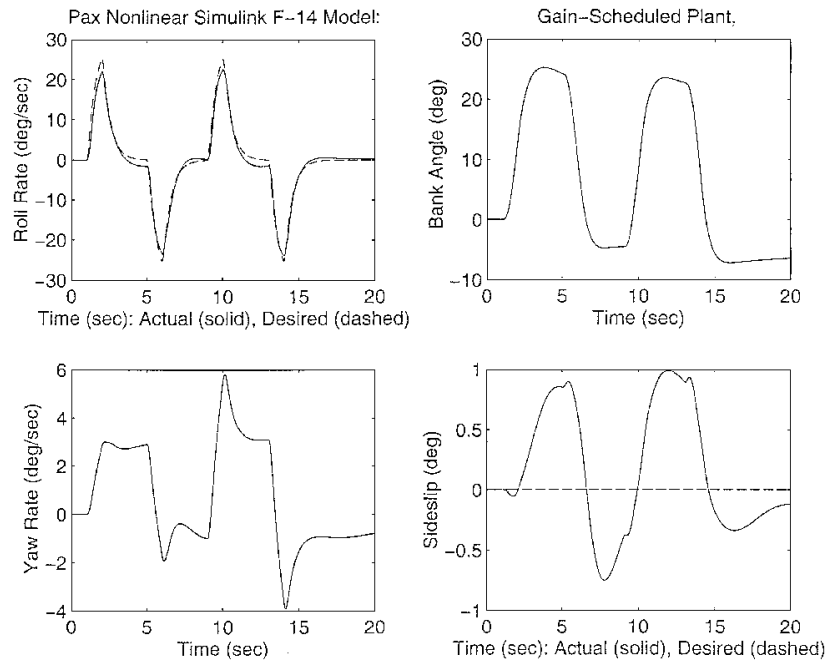

Fig. 9 Lateral stick responses, $p^{`} \phi^{`} r$, and $\beta$.

because of the variation in magnitude and sign of $W_{\text {sr }}(\alpha)$ as a function of $\alpha$. Finally all deflection magnitude and rate limits have been met. Figures 9 and 10 show that in this simulation the sideslip is small and in the adverse direction, obviating the need for stick/pedal coordination with pure lateral stick commands. Sideslip tracking is slightly worse at low $\alpha$ and improves at high $\alpha$ because of the fact that at low $\alpha$ the focus was on reducing the roll-sideslip cross coupling and not on sideslip tracking. This however is consistent with the HQ priorities.

\section{Piloted Simulations}

The controller design discussed in this paper has not been tested in the Manned Flight Simulator at Patuxent River. An earlier version of the present design was tested on 28 August 1996 by an F-14 test pilot, and the observed deficiencies in handling qualities motivated the improved design discussed here. The main difference in the designs is that in the earlier design no attempt was made to compensate for the proverse sideslip tendency at low angles of attack, i.e., the feedthrough weights $W_{\mathrm{ss}}$ and $W_{\mathrm{sr}}$ were not incorporated. For purpose of comparison, the stick response of the earlier design at $\alpha=2$ deg is shown in Fig. 11. The response shows a
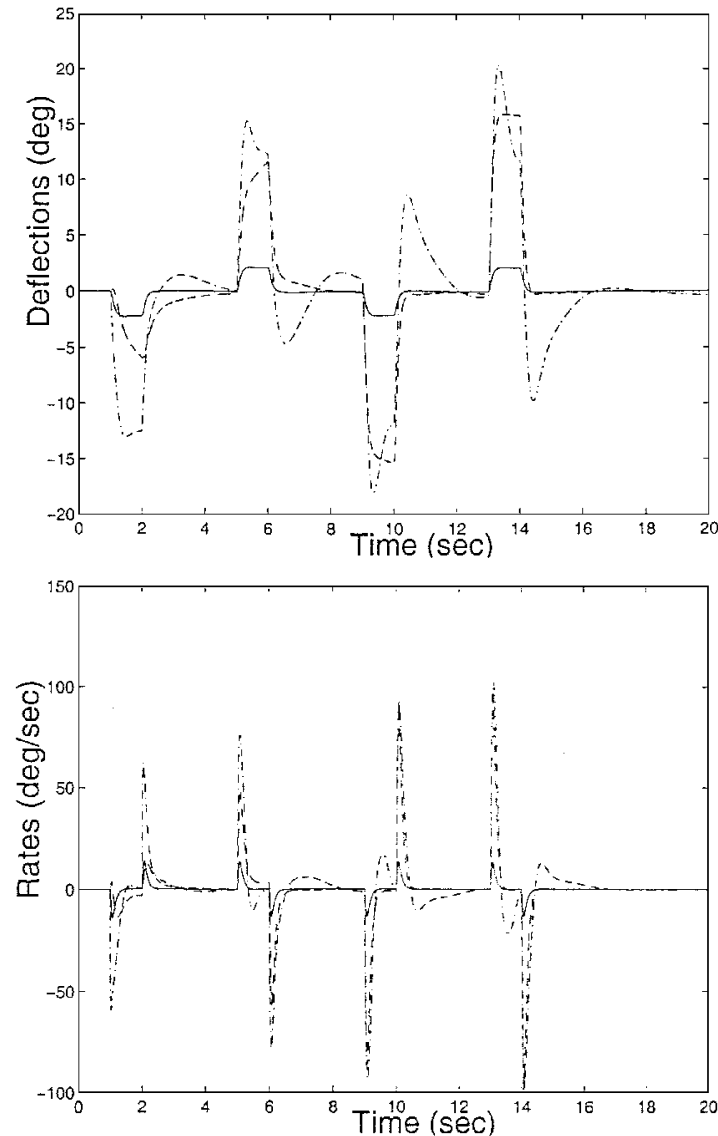

Fig. 10 Lateral stick actuator deflection and deflection rates: differential stablizer $(-)$, rudder $(---)$, and differential spoiler $(-\cdot-)$.

significant amount of proverse sideslip buildup at low angles of attack. This proverse tendency manifested itself in the flight simulations as well and motivated the following comments from the test pilot:

1) "At high speeds [this corresponds to the $\alpha=2$-deg flight condition], $180 \mathrm{kn}$, there is a large amount of proverse sideslip and yaw, i.e., the nose of the aircraft leads. This requires the pilot to lead the heading angle by a few degrees.' 

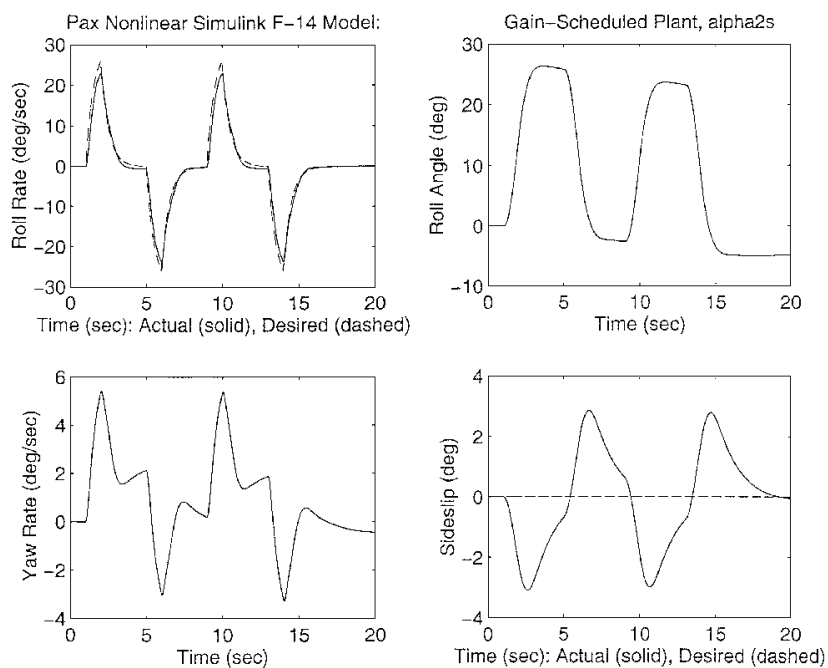

Fig. 11 Roll response of the earlier control design.

2) "The proverseyaw is not a problem when the aircraft is on speed [this corresponds to the $\alpha=10.5$-deg flight condition], though the pilot needs to command smooth inputs since aggressive inputs will introduce more sideslip."

3) "I would give this controller a Cooper-Harper ${ }^{15}$ rating of 4 [which is, by definition, minor deficiencies, moderate pilot compensation] for doing the offset landing task because of the amount of pilot compensation required."

Thus, in summary, the main deficiency of the earlier design was the proverse sideslip tendency at low angles of attack. The present controller eliminates the proverse sideslip tendency and achieves as good roll-rate tracking as the earlier design. It is therefore expected that the present design will require significantly less pilot compensation during powered approach. Because of funding and time constraints, the redesigned gain-scheduledLFT controller was unable to be tested in piloted simulations.

\section{Conclusions}

The variation in F-14 lateral dynamics with angle of attack necessitates a gain-scheduled approach to the lateral control design problem. A linear fractional model of the F-14 lateral dynamics was developed based on four linearized models that represent the dynamics during PA. This linear fractional model was used to design a linear fractional transformation gain-scheduled controller that schedules on angle of attack. In addition to achieving the desired stick/ rudder HQs, special attention was paid to eliminating the natural proverse tendency of the aircraft at low angles of attack. The design has been verified through nonlinear simulations of the present controller, as well as via comparison with pilot-in-the-loopsimulations of earlier controller versions.

\section{Appendix: F-14 LFT Model}

The matrices $M_{0}, M_{1}, M_{2}$, and $M_{3}$ that define the F-14LFT model are defined as follows:

$$
\begin{aligned}
& M_{0}=\left[\begin{array}{ccccccc}
-0.1737 & -337.5224 & 1.8344 & 32.1743 & 0.1770 & 0.2154 & 0 \\
0.0038 & -0.3586 & -0.0659 & 0 & -0.0128 & -0.0254 & -0.0021 \\
-0.0114 & 0.5403 & -2.6185 & 0 & -0.1009 & 0.0148 & -0.0989 \\
0 & -0.0014 & 1.0000 & 0 & 0 & 0 & 0 \\
0.1708 & 0 & 0 & 0 & 0 & 0 & 0 \\
-0.0005 & -0.9958 & 0.0019 & 0.0933 & 0.0005 & 0.0006 & 0 \\
0 & 0 & 57.2958 & 0 & 0 & 0 & 0 \\
0 & 57.2958 & 0 & 0 & 0 & 0 & 0 \\
0 & 0 & 0 & 57.2958 & 0 & 0 & 0 \\
-0.0049 & 0.0065 & 0.0796 & 0 & 0.0074 & 0.0045 & 0.0007
\end{array}\right] \\
& M_{1}=\left[\begin{array}{ccccccc}
0.0072 & 14.2143 & 4.9970 & -0.0005 & -0.0162 & -0.0159 & 0 \\
-0.0002 & 0.0158 & -0.0145 & 0 & 0.0003 & 0.0026 & 0.0005 \\
-0.0008 & -0.0018 & 0.1465 & 0 & 0.0071 & -0.0001 & 0.0088 \\
0 & 0.0179 & 0 & 0 & 0 & 0 & 0 \\
0.0071 & 0 & 0 & 0 & 0 & 0 & 0 \\
-0.0000 & 0.0000 & 0.0174 & 0.0050 & -0.0000 & -0.0000 & 0 \\
0 & 0 & 0 & 0 & 0 & 0 & 0 \\
0 & 0 & 0 & 0 & 0 & 0 & 0 \\
0 & 0 & 0 & 0 & 0 & 0 & 0 \\
0.0003 & -0.0015 & -0.0035 & 0 & -0.0006 & -0.0003 & -0.0001
\end{array}\right] \\
& M_{2}=\left[\begin{array}{ccccccc}
-0.0002 & -0.3480 & -0.0995 & -0.0048 & 0.0005 & 0.0005 & 0 \\
0.0000 & -0.0010 & 0.0001 & 0 & 0.0001 & -0.0002 & -0.0001 \\
-0.0001 & 0.0020 & -0.0026 & 0 & -0.0002 & -0.0002 & -0.0007 \\
0 & 0 & 0 & 0 & 0 & 0 & 0 \\
0 & 0 & 0 & 0 & 0 & 0 & 0 \\
0.0000 & 0.0001 & 0 & -0.0001 & 0.0000 & 0.0000 & 0 \\
0 & 0 & 0 & 0 & 0 & 0 & 0 \\
0 & 0 & 0 & 0 & 0 & 0 & 0 \\
0 & 0 & 0 & 0 & 0 & 0 & 0 \\
-0.0000 & 0.0000 & 0.0001 & 0 & 0.0000 & 0.0000 & 0.0000
\end{array}\right]
\end{aligned}
$$




$M_{3}=1.0 e-04 \cdot\left[\begin{array}{ccccccc}0 & 0 & 0 & 0 & 0 & 0 & 0 \\ -0.0093 & 0.3453 & 0.5700 & 0 & -0.0591 & 0.0444 & 0.0276 \\ 0.0447 & -0.6561 & 0 & 0 & 0 & 0.0964 & 0.2674 \\ 0 & 0 & 0 & 0 & 0 & 0 & 0 \\ 0 & 0 & 0 & 0 & 0 & 0 & 0 \\ 0 & 0 & 0 & 0 & 0 & 0 & 0 \\ 0 & 0 & 0 & 0 & 0 & 0 & 0 \\ 0 & 0 & 0 & 0 & 0 & 0 & 0 \\ 0 & 0 & 0 & 0 & 0 & 0 & 0 \\ 0 & 0 & 0 & 0 & 0 & 0 & -0.0049\end{array}\right]$

\section{Acknowledgments}

This work was performed by MUSYN, Inc., under Contract N00421-94-R-0094 from the Naval Air Warfare Center at Patuxent River, Maryland. We would like to thank Dale Enns for suggesting the $p / \phi$ blending to us.

\section{References}

${ }^{1}$ Durand, T. S., and Wasicko, R. J., "Factors Influencing Glide Path Control in Carrier Landing," Journal of Aircraft, Vol. 4, No. 2, 1965, pp. 146-158.

${ }^{2}$ Nordwall, B. D., "U.S. F-14s to Get Digital Controls," Aviation Week and Space Technology, Vol. 147, No. 19, 1996, p. 64.

${ }^{3}$ Hughes, D., "Navy Begins Testing of F-14 Digital Controls," Aviation Week and Space Technology, Vol. 148, No. 35, 1995, p. 22.

${ }^{4}$ Denham, J., Liebler, S., and Renfrow, J., "F-14 Flight Control Law Design, Verification and Validation Using Computer Aided Engineering Tools," Naval Air Warfare Center Rept. Patuxent River, MD, March 1995.

${ }^{5}$ Balas, G. J., Packard, A. K., McCloskey, R. T., Renfrow, J., and Mullaney, C., "Design of a Controller for the F-14 Aircraft Lateral-Directional Axis During Powered-Approach," Journal of Guidance, Control, and Dynamics, Vol. 21, No. 6, 1998, pp. 899-908; also AIAA Paper 96-3781, Aug. 1996.

${ }^{6}$ Balas, G. J., and Doyle, J. C., "Robustness and Performance Tradeoffs in Control Design for Flexible Structures," IEEE Transactions on Control Systems Technology, Vol. 2, No. 4, 1994, pp. 352-361.

${ }^{7}$ Balas, G. J., Doyle, J. C., Glover, K., Packard, A. K., and Smith, R. "The $\mu$ Analysis and Synthesis Toolbox," MUSYN and The Mathworks,

The Mathworks Inc., Natick, MA, 1991, 1993.

${ }^{8}$ Balas, G. J., and Packard, A. K., "The Structured Singular Value $(\mu)$ Framework," CRC Controls Handbook, CRC Press, Boca Raton, FL, 1996, Sec. 2.3.6.

${ }^{9}$ Doyle, J., Wall, J., and Stein, G., "Performance and Robustness Analysis for Structured Uncertaintly," IEEE Conference on Decision and Control, Vol. 1, Inst. of Electrical and Electronics Engineers Press, Piscataway, NJ, 1982 , pp. 629-636.

${ }^{10}$ Packard, A. K., "Gain Scheduling via Linear Fractional Tranemormations," System and Control Letters, Vol. 22, No. 2, 1994, pp. 79-92.

${ }^{11}$ Balas, G. J., Packard, A. K., and McCloskey, R. T., "F-14 Robust Control Analysis and Synthesis," Naval Air Warfare Center, Phase 1 Final Rept., Contract N00421-94-R-0094, Patuxent River, MD, Sept. 1995.

${ }^{12}$ Belcastro, C., "Parametric Uncertainty Modeling: An Overview," Proceedings of the American Control Conferences, Vol. 2, Inst. of Electrical and Electronics Engineers Press, Piscataway, NJ, 1998, pp. 992-996.

${ }^{13}$ Lambrechts, P., Terlouw, J., Bennai, S., and Steinbuch, M., "Parametric Uncertainty Modeling Using LFTs," Proceedings of the American Control Conference, Vol. 1, Inst. of Electrical and Electronics Engineers Press, Piscataway, NJ, 1993, pp. 267-272.

${ }^{14}$ Cockburn, J. C., "Linear Fractional Representations of Systems with Rational Uncertainty," Proceedings of the American Control Conference, Vol. 2, Inst. of Electrical and Electronics Engineers Press, Piscataway, NJ, 1998, pp. 1008-1012.

${ }^{15}$ Cooper, G. E., and Harper, R. P., "The Use of Pilot Rating in the Evaluation of Aircraft Handling Qualities," NASA-TN-D-5153, May 1969. 